\title{
Application of Series Formula Method in Optimization Arrangement of Webs
}

\author{
Qiaozhi Sang1, Siwen Li1 ${ }^{1}$, Shuqi Wang2 ${ }^{2}$ Dianxuan Gong ${ }^{3 *}$ \\ ${ }^{1}$ Yisheng College, North China University of Science and Technology, Tangshan, China \\ ${ }^{2}$ College of Architectural Engineering, North China University of Science and Technology, Tangshan, China \\ ${ }^{3}$ College of Science, North China University of Science and Technology, Tangshan, China \\ Email: *dxgong@ncst.edu.cn
}

How to cite this paper: Sang, Q.Z., Li, S.W., Wang, S.Q. and Gong, D.X. (2018) Application of Series Formula Method in Optimization Arrangement of Webs. World Journal of Engineering and Technology, 6, 651-660.

https://doi.org/10.4236/wjet.2018.63041

Received: May 2, 2018

Accepted: July 29, 2018

Published: August 1, 2018

Copyright (C) 2018 by authors and Scientific Research Publishing Inc. This work is licensed under the Creative Commons Attribution International License (CC BY 4.0).

http://creativecommons.org/licenses/by/4.0/

\begin{abstract}
The expressions of the internal forces of the webs under the vertical loads of the simply supported beam type trusses with vertical and horizontal webs and without vertical webs are studied through the mathematical formula method. The variations of internal forces under different angles and spacings of the webs are simulated. The law of the optimal arrangement of the webs of the parallel-string simple-beam truss is obtained: under the condition that the rigidity of the rod is allowed, the form of no vertical web-type truss and reducing the span distance and inclination of the side span are advisable, which can save materials and reduce the weight as well. This method can be applied to the calculation of internal forces for arbitrary loads and truss forms.
\end{abstract}

\section{Keywords}

Series Formula Method, Beam Truss, Optimum Arrangement of Webs

\section{Introduction}

The domestic and foreign methods for calculating the internal force of a simple truss plane in statically determinate planes are mainly numerical solutions and graphical methods at present. To calculate the internal force using the numerical solution method, it is necessary to "truncate" the trusses and list the balance equations. Generally, the truncated rods can be obtained when they do not exceed 3 [1]. This method is widely used for calculating the internal forces of a simple truss, but it is very cumbersome to use for complex and multi-node trusses. In the design, the graphic method is usually used, but the designer must have certain experience and a more accurate drawing level in the drawing of interaction diagrams of internal forces. Liu Yuehua solved the internal forces of trusses by 
obtaining two unknown forces at a node combined with AUTO CAD drawing tools based on the graphic method [2]. M. S. Kechum proposed an exponential internal force method, in which the vertical force components of the rod represent its internal force in the form of a trigonometric function, and the internal forces of the truss members are quickly calculated [3]. For the fasting staggered truss structure, Zhou Xuhong et al. introduced the truss rotational stiffness and transfer coefficient to give the formula for internal forces of the members, which was in good agreement with the results of ANSYS analysis [4]. The mathematical formula method is a simple and applicable method combining the index internal force method with the traditional cross section method, the truss regarded as an ideal one, which is composed of the calculation sections between the left end and the right end arranged in series, The equation derived from the section method containing only one unknown based on the principle of the virtual work method, is used to calculate the internal force of a simple abdominal truss [5].

\section{Truss and Rod Parts Classification}

Truss refers to a lattice-based lattice structure consisting of straight rods connected to each other at their ends. The simplest form of a simple abdominal truss is a triangle formed by three rods hinged to each other at the ends of the rods. Under the action of a load, the inherent shape of the truss is not changed except for a slight elastic deformation. Each time two poles are accessed, various types of stable trusses can be obtained. The truss joined by this method is called a simple abdominal truss. Among them, the corresponding nodes of the upper and lower chords, in addition to the oblique web members, also have a vertical web-joint truss called a vertical web truss; a diagonal web only truss is called a vertical web truss. In a truss, there is a vertical web between some of the joints, and the other truss without a vertical web is called a hybrid web truss.

The member in a truss between the two upper chords is called the upper chord, denoted as $O$, the member between lower chord nodes is referred to as $U$. The rod connecting the upper and lower strings is called the web rod, Tilt rods that are inclined to the right are called ascending diagonal webs, which are represented by $D^{u p}$, otherwise by $D^{\text {down }}$. The middle diagonal rods of two adjoining ascending cantilever rods are called ascending vertical web rods denoted by $V^{u p}$ the corresponding descending vertical web rods are $V^{\text {down }}$; when the upper rod rods are bent at the upper end of a single vertical web rod, the single vertical rod is called “个” rod represented by $V_{o}$, if the vertical and horizontal rods are diagonal rods, they are called $V_{o}^{\prime \prime}$. When the upper chord is penetrated at the upper end of a single vertical bar, the single vertical bar is called a T-shaped vertical bar denoted by $V_{t}$, it turns into T diagonal rods $V_{t}^{\prime \prime}$ when the vertical rods are sloping. The inverted vertical and rods and inverted diagonal rods are denoted by $V_{u}$ and $V_{u}^{\prime \prime}$ correspondingly; the inverted 
T-shaped vertical abdominal rods and inverted T-shaped diagonal abdominal rods are presented by $V$ and $V^{\prime \prime}$. The definition of each specific bar is shown as follows (Figure 1).

\section{Introduction to Series Formula Method}

\subsection{Method Description}

The definition of the names of the rods and the symbols of the formulas are shown in Figure 1. The upper (lower) chord of the truss is called the upper chord, and the upper (lower) one between $x$ calculation sections is called the upper (lower) chord. The slanted web that slopes to the right in this section is called ascending slant rod, and that slanting in the left is called descending slant rod. The middle vertical and horizontal webs of the adjacent upward-rising sloping rods are known as ascending vertical webs, and the middle vertical webs of two adjacent downward webs are called descending vertical webs. The interval between adjacent webs is called the calculation interval denoted by $x$. The numbering rule of interval is:

Trusses with vertical rod is arranged in natural number sequence (0), 1, 2, 3, etc., while for trusses without a vertical rod, it is in order of arithmetic series (0), $1,1.5,2,2.5,3$ etc. when the first rod is an ascending oblique web, of arithmetic series (0), 0.5, 1, 1.5, 2, 2.5 ect., when the first rod declines. In that case, when arranged sequentially from left to right in order of arithmetic progression, the internal force equation deducted by the section method can be simplified according to the sum formula of arithmetic series, as we all know $S_{n}=n a_{1}+[n(n-1) / 2] d$.

The ultimate expression of internal forces is simple and has wider applicability, which is capable to calculate any rod or node displacement of the truss.

\subsection{Derivation of Series Formula for Internal Force}

The internal force $O_{x}$ of upper chord in the $X^{\text {th }}$ inter-section: using the cross section method [6], take the left side of section a-a as the spacer, the lower node as the mausoleum, then $\sum M_{X 0}=0$

$$
\begin{gathered}
M_{u x}+O_{x} r_{o x}=0 \\
O_{x}=-M_{u x} \frac{i_{o c x}}{i_{o b x} h_{x}}
\end{gathered}
$$

The lower chord internal $U_{x}=M_{o x} \frac{i_{o c x}}{i_{o b x} h_{x}}$ force is obtained in the same way as Figure 2 shows:

Ascending oblique web internal force $D_{x}^{u p}$ in the $X^{\text {th }}$ inter-section: take the left end of the section a-a as a spacer, $\sum y=0$, then

$$
\begin{gathered}
Q_{x}+O_{x-1} \frac{1}{i_{o c x-1}}-U_{x} \frac{1}{i_{u c x}}+D_{x}^{u p} \frac{1}{i_{d c x}^{u p}}=0 \\
D_{x}^{u p}=-Q_{x} i_{d c x}^{u p}=-Q_{x} / \cos \theta_{d c x}^{u p}
\end{gathered}
$$




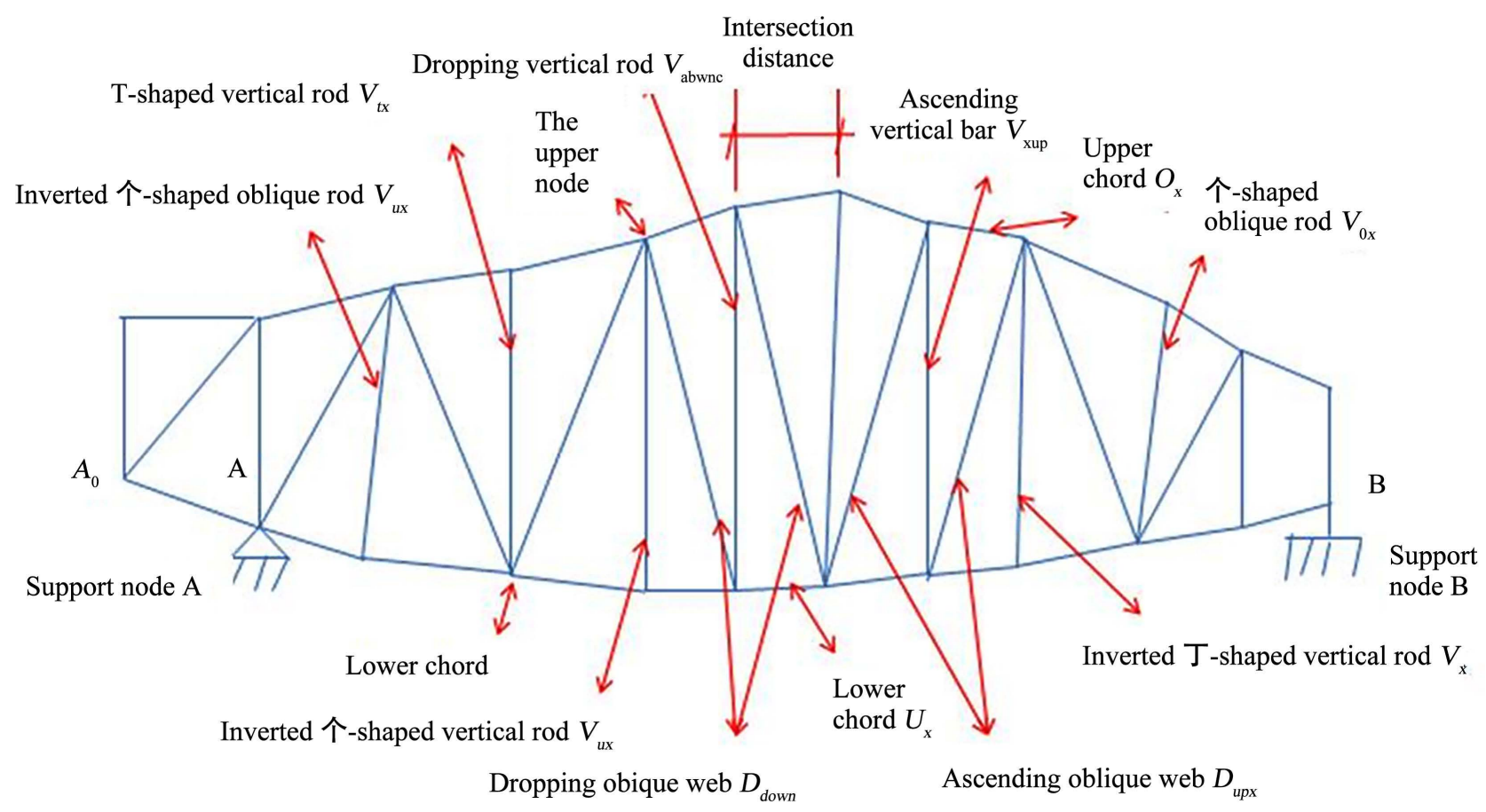

Figure 1. Definition of each bar in a truss.

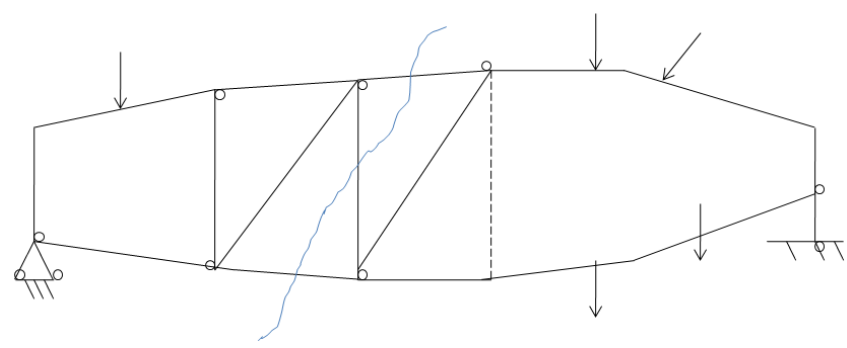

Figure 2. Section selected by section method.

The corresponding internal force of a falling oblique rod is $D_{x}^{\text {down }}=Q_{x}{ }_{d c x}^{\text {down }}=Q_{x} / \cos \theta_{d c x}^{\text {down }}$.

The internal force $V_{x}^{u p}$ of ascending vertical abdominal rod in the $X^{\text {th }}$ inter-section: take the left part of section a-a as a separator [7], $\sum y=0$

$$
\begin{gathered}
Q_{x}+O_{x} \frac{1}{i_{\text {ocx }}}-U_{x} \frac{1}{i_{u c x}}-V_{x}^{u p}=0 \\
V_{x}^{u p}=Q_{x}
\end{gathered}
$$

The internal force of a falling vertical $\operatorname{rod} V_{x}^{\text {down }}=-Q_{x}$ is calculated through the same way.

The internal force of "个” vertical bar $V_{o x}$ and oblique rod $V_{o x}^{\prime \prime}$ : take the separator above section a-a (see Figure 3), $\sum y=0$, then

$$
\begin{gathered}
-O_{x} \frac{1}{i_{o c x}}-O_{x}^{\prime} \frac{1}{i_{o c x}^{\prime}}+p_{x}-V_{o x}=0 \\
V_{o x}=p_{x}, V_{o x}^{\prime \prime}=p_{x} / \cos \theta
\end{gathered}
$$




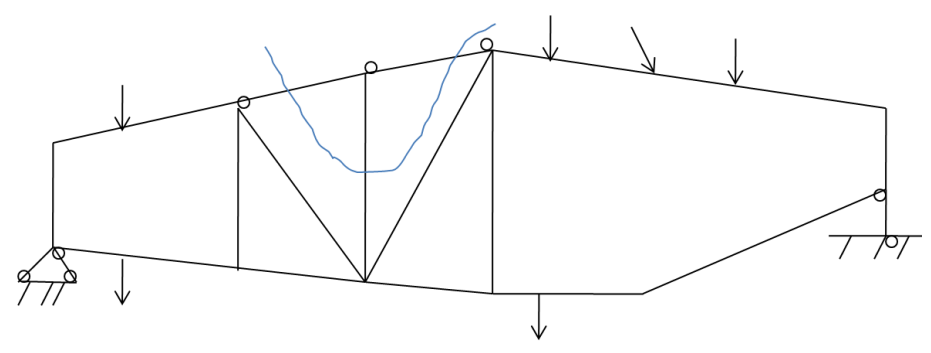

Figure 3. Section selected for “个” vertical bar.

The inner force of inverted “个” vertical rod $V_{o x}$ is

$$
V_{u x}=G_{x}
$$

and that of the inclined one $V_{o x}^{\prime \prime}$ is

$$
V_{u x}^{\prime \prime}=\frac{G_{x}}{i_{g c x}} i_{v c x}
$$

\subsection{Expressions for Internal Forces in a Truss}

3.3.1. The Internal Force of Vertical Truss under the Action of Unit Vertical Node Load (X Stands for the Rod Adjacent to the Left Side of the X-Section)

$$
\begin{gathered}
D_{x}^{u p}=-0.5(n-2 x+1) / \cos \theta \\
D_{x}^{d o w n}=0.5(n-2 x+1) / \cos \theta \\
V_{p x}^{u p}=0.5(n-2 x-1) \\
V_{p x}^{d o w n}=-0.5(n-2 x+1) \\
V_{p o x}=-1 \\
V_{p u x}=0
\end{gathered}
$$

3.3.2. The Internal Force of Truss without Vertical Web under Vertical Load Per Unit

Half-section truss between upper chords

$$
\begin{gathered}
D_{x}^{u p}=\left(R_{A}-x+1.25\right) / \cos \theta \\
D_{x}^{\text {down }}=\left(R_{A}-x+1.25\right) / \cos \theta \\
D_{1}^{u p}=-R_{A} / \cos \theta \\
D_{B x}^{\text {down }}=-R_{B} / \cos \theta
\end{gathered}
$$

Half-section truss between lower chords

$$
\begin{gathered}
D_{x}^{u p}=-\left(R_{A}-x+1\right) / \cos \theta \\
D_{x}^{\text {down }}=\left(R_{A}-x+0.5\right) / \cos \theta \\
D_{0.5}^{\text {down }}=R_{A} / \cos \theta_{0.5}^{\text {down }} \\
D_{B x}^{u p}=-R_{A} / \cos \theta^{u p}
\end{gathered}
$$




\section{The Arrangement of Rods}

The arrangement of the webs should be such that the truss structure is simple and the force is reasonable. As far as possible, the long rods are subjected to tension and the short one are compressed. The inclined web angles are generally from $30^{\circ}$ to $60^{\circ}$. The external load should act on the node to avoid the local bending moment of the chord due to the inter-node load. This paper investigates the variation of internal forces of the simple-supported beam truss with vertical and radial webs and vertical and horizontal rods under three-dimensional vertical loads with the arrangement of the webs. Among them, there are vertical abdominal truss arrangements of the abdominal system is divided into the left half of the rise and the right half of the decline, the left half of the decline and the right half of the rising, falling and ascending alternate arrangement, ascending and descending Formed into "eight"-shaped layout; no vertical web beam simple beam truss according to the abdominal layout of the winding head joints for the half pitch, the lower end of the chord between the half pitch, the number of winding internodes greater than the lower chord joint The trusses with the number and the number of inter-chords less than the number of inter-chords are shown in Figure 4 and Figure 5.

\section{Application of Series Formula Method in Arranging Truss Webs}

Based on the calculation formula given above, the internal forces of the rods are changed with the inclination of the rods and the spacing between the webs, respectively, so that the angle between the webs and the vertical direction changes from $30^{\circ}$ to $60^{\circ}$, and the spacing ranges from $10 \mathrm{~cm}$ to $50 \mathrm{~cm}$. Change; Each curve represents a specific position in the truss. The position of the rod varies from bottom left to right depending on the truss arrangement. It varies from bottom to top or top to bottom. Take a left-rise, right-fall truss as an example:

The left end is the ascending type abdominal rod, the internode position $x<\frac{n}{2}$, so with the position of the abdominal rod from the left end to the mid-span, the pressure $D_{x}^{u p}$ is gradually reduced. In the midspan, it is 0 , that is, the end of the abdominal rod is greater than the middle abdominal rod; The shape of the rod parallel trusses does not conform to the shape of the bending moment diagram, so the force is poor, that is, the internal force of the chord is not even and the internal force of the web is slightly larger. The web changes with inclination from $30^{\circ}$ to $60^{\circ}$, and the internal pressure decreases. The lateral spandrel tends to converge when the tilt angle approaches $60^{\circ}$, while the internal force of the intermediate span web changes most sensitively around $60^{\circ}$; At a certain angle, with the increase in the number of webs, the internal pressure also increases because the corresponding webs of the joints gradually move toward the span along the distance between the nodes, close to the force transmission path, and the internal force and spacing of the webs become Linear change. The 

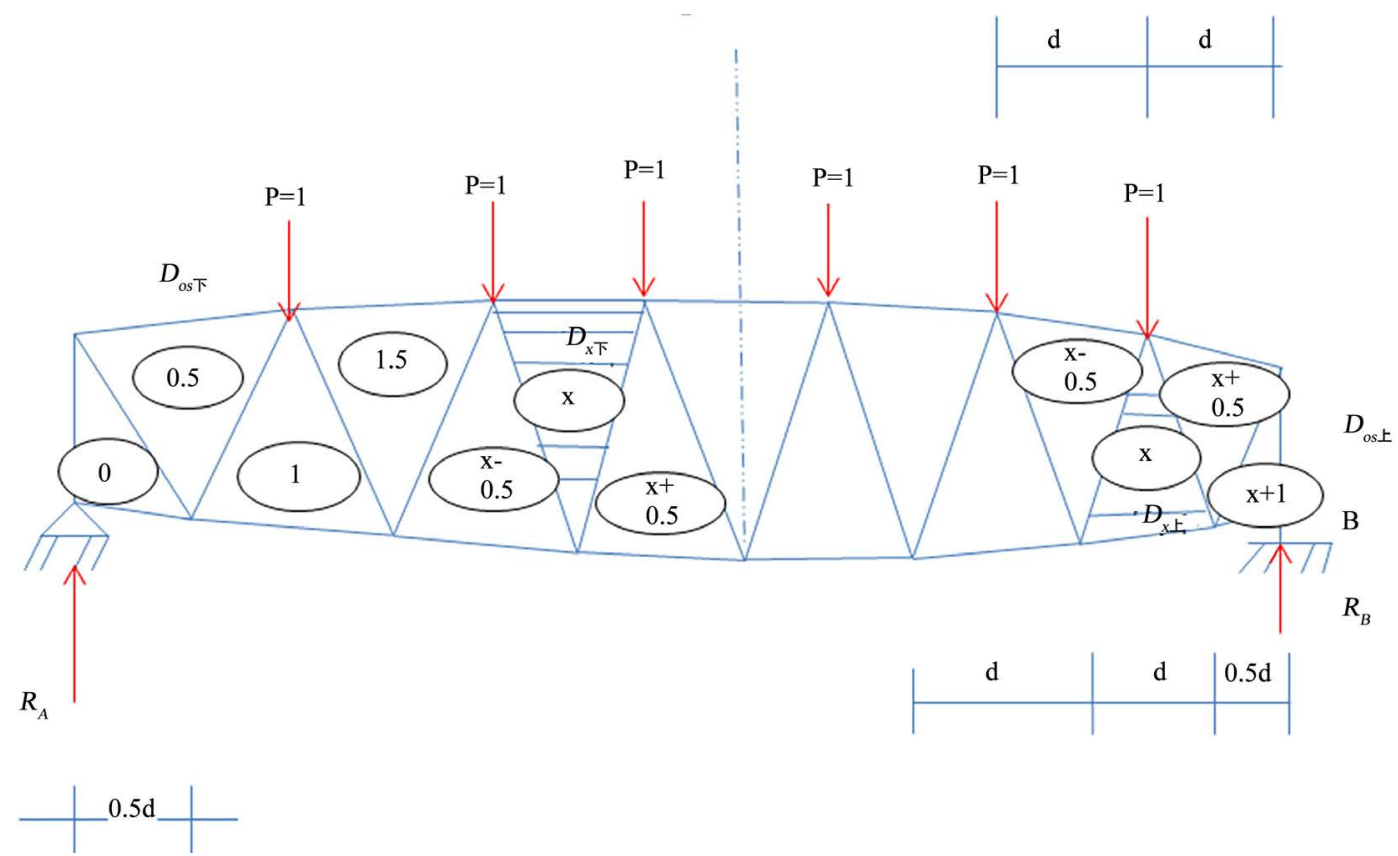

Figure 4. Truss with half-section spacing at the lower end of the chord.

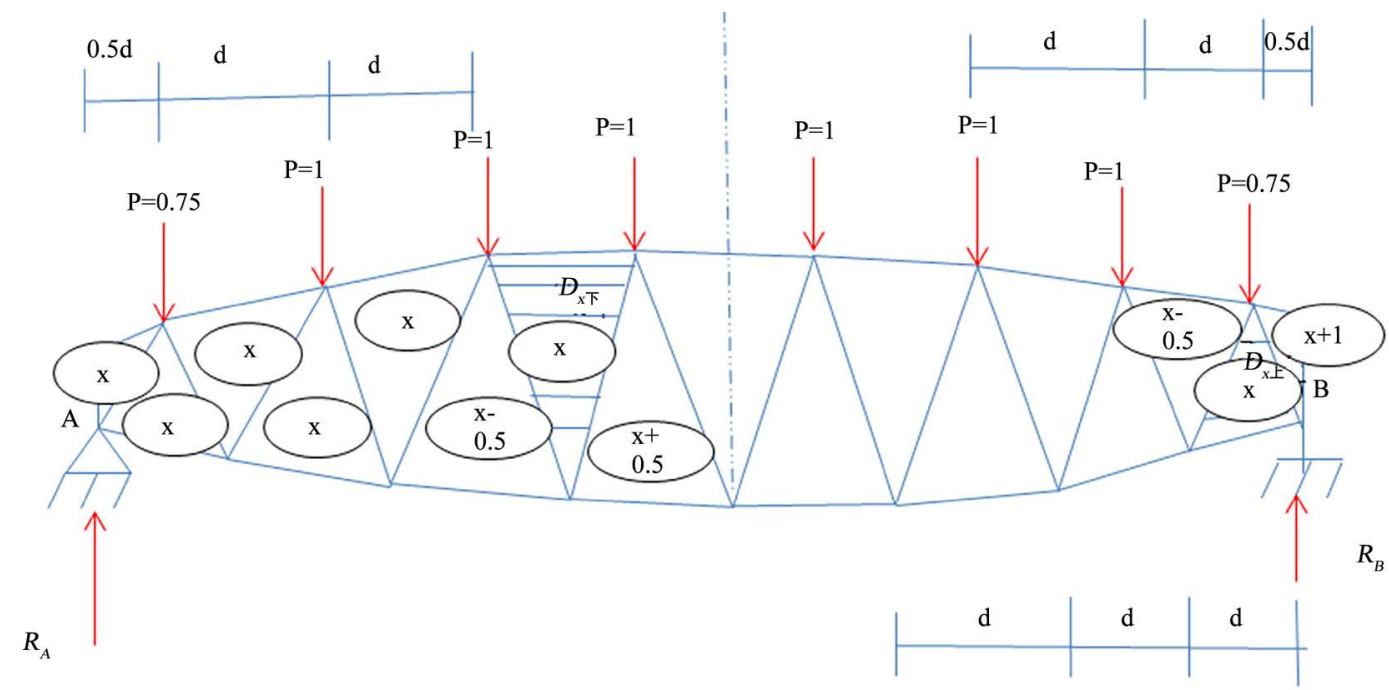

Figure 5. Truss with half-section spacing at the higher end of the chord.

descending abdominal rod changes completely symmetrically with the rising trend of the corresponding internode rising diagonal rod. The internal forces of the lateral spanning rod are most sensitive to changes in the dip angle. For every $6^{\circ}$ increase near $60^{\circ}$, the pressure on the web increases by approximately 20 (see Figure 6).

The tension of the upright vertical web is located on the left span, and the internal force varies steadily with the pitch of the joints. The pull force of the web between the joints decreases with the increase of the pitch, because the internodes increase with the pitch between the nodes. While gradually moving across the middle, but from the truss system, its internal force amplitude showed a 
downward trend. The right cross-descent vertical bar is completely reversed symmetrical with the ascending change (see Figure 7).

For a parallel string truss without a vertical web, the ascending web is under pressure and falling, and the change is basically the same as that of a truss. The number of rods and the number of nodes in the vertical web-type truss system is also small. The two web members in the same node pull one pressure, and can well withstand the change load.

\section{Conclusions}

This paper discusses the variation of the internal force with the pitch angle and pitch of the truss system of the two kinds of truss systems with vertical and horizontal webs. In order to avoid the local bending moment of the inter-chord chords, the unit load is assumed to act on each of the upper and lower nodes. In order to simplify the calculation and facilitate the discussion, the simple-supported beam truss with parallel strings (left-wing rising right-side descending arrangement) as an example, the following results are given:
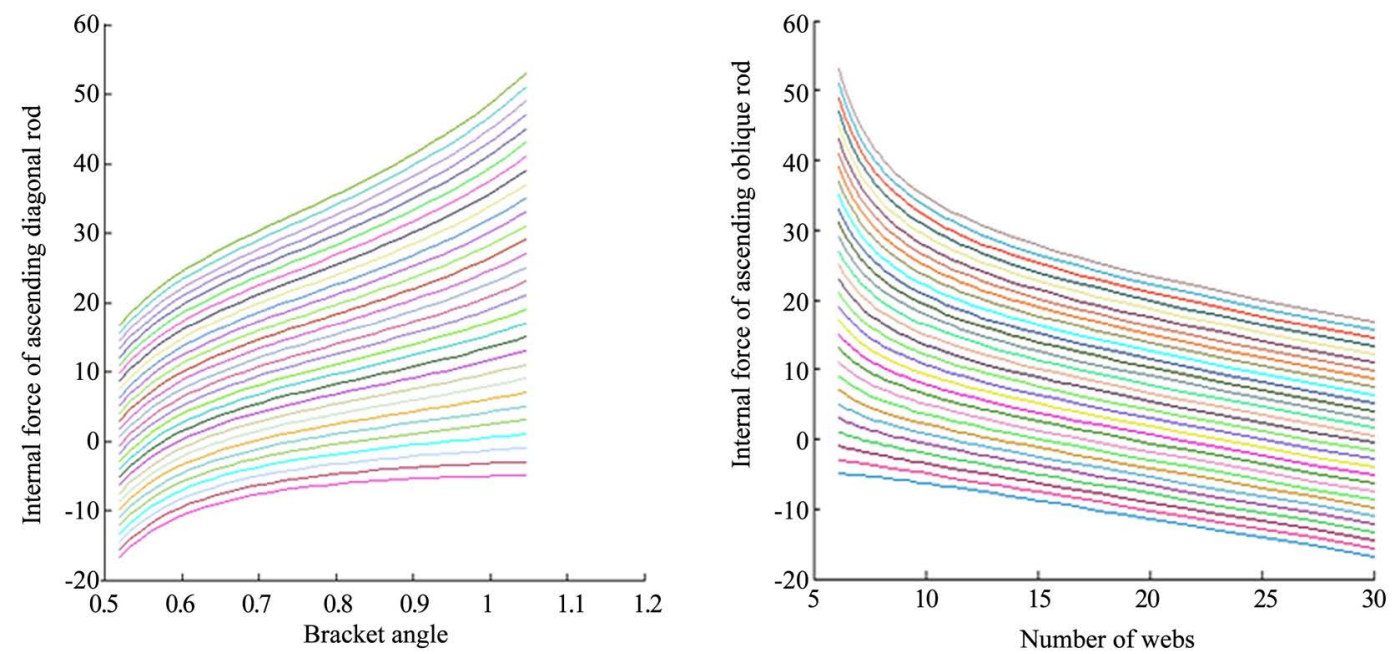

Figure 6. Ascending oblique rod internal force varies with dip angle and quantity.
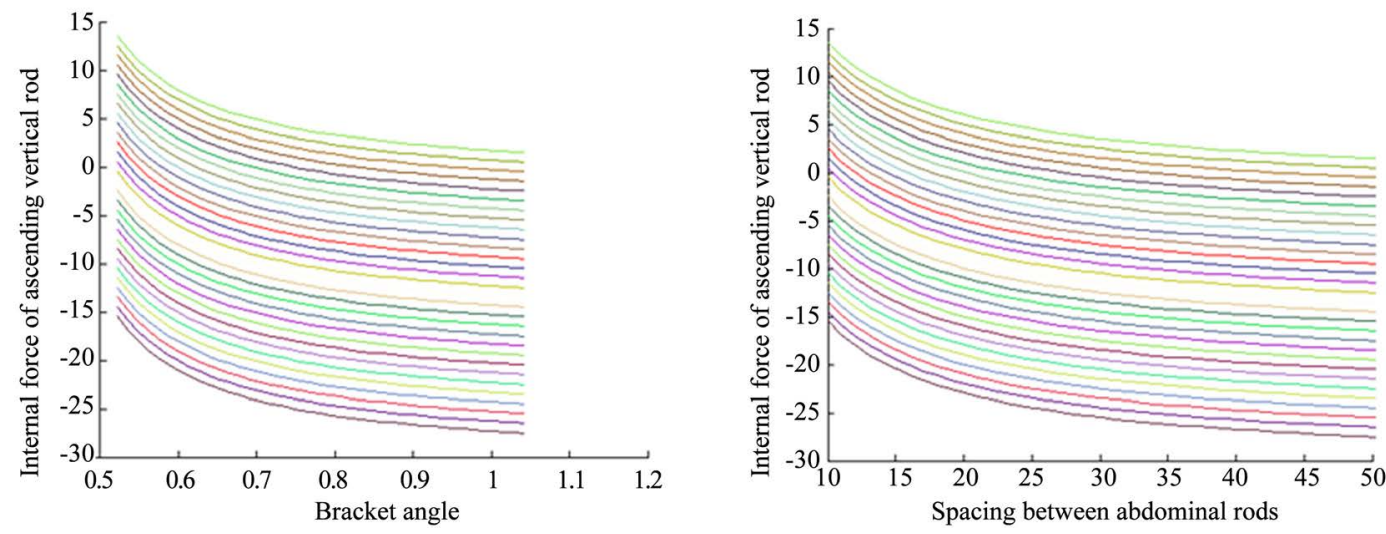

Figure 7. Ascending vertical rod internal force varies with dip angle and quantity. 
Because the intermediate cross-web rod is far from the force transmission path, in order to save material and reduce its own weight, the number of arranged cross-middle web members can be reduced; when the angle between the middle web member and the vertical direction is about $60^{\circ}$, its internal force has little sensitivity to the angle. The force is stable, so it is possible to arrange the web about $60^{\circ}$ near the middle position; the internal force of the web-span web increases with the tilt angle, and its sensitivity also gradually increases. Therefore, the web tilt angle can be reduced to about $30^{\circ}$ at the side span. It is appropriate.

The corresponding webs of a particular node will reduce as the pitch between the nodes decreases, and the rod moves toward the side span or the mid-span, and the internal force received by the rod increases or decreases. However, from the point of view of the truss system, the magnitude of the internal force as a whole showed a downward trend. Therefore, when the webs are properly increased, external loads can be evenly distributed to the rods. In order to reduce the self-weight, a reasonable arrangement may be to reduce the gap between the spans and to increase the pitch between the webs.

The number of rods and the number of nodes in the vertical web-type truss system are also small. The two web members in the same node pull one pressure, and can well withstand the change load. Therefore, it is advisable to use a vertical truss-type truss when the stiffness of the member allows.

\section{Acknowledgements}

This project is supported by National Natural Foundation of China (11301120, 11601151), National Natural Foundation of Hebei Province (A2015209189) and Young Talents Support Project of Hebei Province.

\section{Conflicts of Interest}

The authors declare no conflicts of interest regarding the publication of this paper.

\section{References}

[1] Wu, M. and Ding, K.W. (2013) Discussion on the Calculation Method of Truss Internal Forces. Mechanics and Practice, 35, 82-84.

[2] Liu, Y.H. and Li, L.Y. (1999) Using AUTOCAD to Solve Truss Internal Forces. Sichuan Building Science, No. 1, 57-58.

[3] Kechum, M.S. and Feng, C.K. (1984) Simple Calculation of Internal Forces of Truss Members. Railway Construction, No. 3, 34-35.

[4] Zhou, X.H. and Chou, Z.S. (2004) Calculation of Internal Force and Lateral Displacement of Staggered Truss Structure under Horizontal Load. Journal of Building Structures, No. 4, 66-71.

[5] Du, K. (1987) Calculation of the Axial Force of a Statically Determinate Plane Beam Truss Using the Series Formula Method. Industrial Construction, No. 6, 29-34.

[6] Zhou, X.H. and Zhou, S. (2004) Calculation of Internal Force and Lateral Shift of Staggered Truss Structure under Horizontal Load. Journal of Building Structures, 
No. $4,66-71$.

[7] Zhou, X.H., Zhou, S. and Huang, X.X. (2004) Calculation of Internal Forces of Staggered Truss Structures under Vertical Loads. Engineering Mechanics, No. 3, 25-30. 